\title{
Regional amyloid accumulation and cognitive decline in initially amyloid-negative adults
}

Michelle E. Farrell, PhD, Xi Chen, MS, Melissa M. Rundle, PhD, Micaela Y. Chan, PhD, Gagan S. Wig, PhD, and Denise C. Park, PhD

Neurology ${ }^{\circledR}$ 2018;91:e1809-e1821. doi:10.1212/WNL.0000000000006469

\author{
Correspondence \\ Dr. Farrell \\ mfarrell13@ \\ mgh.harvard.edu
}

\section{MORE ONLINE}

- CME Course

NPub.org/cmelist

\section{Methods}

One hundred twenty-six initially amyloid-negative, cognitively normal participants (age 30-89 years) were included from the Dallas Lifespan Brain Study who completed florbetapir PET and a cognitive battery at baseline and 4-year follow-up. Standardized uptake value ratio (SUVR) change was computed across 8 bilateral regions of interest. Using general linear models, we examined the relationship between change in global and regional SUVR and change in episodic memory, controlling for baseline SUVR, baseline memory, age, sex, education, and APOE status.

\section{Results}

In initially amyloid-negative adults, we detected a regionally specific relationship between declining episodic memory and increasing amyloid accumulation across multiple posterior cortical regions. In addition, these amyloid-related changes in memory persisted when we focused on middle-aged adults only and after controlling for atrophy in global cortical, hippocampal, and Alzheimer disease signature cortical volume.

\section{Conclusion}

Our results indicate that assessing regional changes in amyloid, particularly in posterior cortical regions, can aid in the early detection of subclinical amyloid-related decline in episodic memory as early as middle age. Future research incorporating tau and other markers of neurodegeneration is needed to clarify the sequence of events that lead to this early, subclinical memory decline. 


\section{Glossary}

AD = Alzheimer disease DLBS = Dallas Lifespan Brain Study; GLM = general linear model $;$ LPFC = lateral prefrontal cortex; PCC = posterior cingulate cortex; ROI = region of interest; SUVR = standardized uptake value ratio.

The success of antiamyloid therapies aimed at halting Alzheimer disease $(\mathrm{AD})$ may rely on intervention at the earliest sign of amyloid, before downstream consequences on neurodegeneration and cognitive decline. ${ }^{1}$ Unfortunately, relatively little is known about the earliest stages of amyloid deposition because initial deposits often fall below study-specific thresholds for reliably differentiating amyloid from noise with PET imaging ${ }^{2,3}$ and are considered "amyloid negative." An important recent PET study ${ }^{4}$ found that by leveraging longitudinal data in individuals classified as amyloid negative at baseline, it was possible to detect an early synchrony between increasing amyloid burden and declining memory.

The present study examines further the early relationship between increasing amyloid burden and concurrent cognitive decline over 4 years in initially amyloid-negative adults from the Dallas Lifespan Brain Study (DLBS). Autopsy ${ }^{5}$ and PET evidence $^{6-9}$ indicates that initial amyloid deposits aggregate in a more focal manner compared to the more diffuse later patterns often observed in amyloid-positive individuals. Hence, we examined whether a regional approach may optimize detection of early amyloid deposits and its cognitive consequences in adults typically labeled as amyloid negative. Furthermore, because amyloid is known to start depositing as early as middle age, ${ }^{10,11}$ additional analyses focus on a subsample of adults 30 to 59 years of age as a potential early window into $\mathrm{AD}$ pathologic progression. Finally, to better understand the early sequence of events in the $\mathrm{AD}$ pathologic cascade, we assessed whether structural changes might drive an early relationship between amyloid and cognitive decline.

\section{Methods}

\section{Participants}

This study included all DLBS participants who completed amyloid PET scans, structural MRI scans, and a cognitive battery at baseline and 4-year follow-up. Of 288 participants eligible to return, 178 returned (62\%). Reasons for nonreturns included the following: 8 were deceased, 30 were in poor health, 23 were not interested, and 49 were lost to follow-up. Of those who returned, 36 were excluded (19 because of a change in PET scanner at follow-up, 11 missing MRI/poor MRI image quality, and 6 poor PET-MRI coregistration), leaving a final sample of 142 participants. Those retained $(n=$ 142) did not differ significantly from those excluded $(n=146)$ as a function of age, baseline standardized uptake value ratio (SUVR), years of education, sex, or APOE carrier status (all $p$ > 0.10 ). Analyses in the current study focused on the amyloidnegative subsample $(n=126)$, with description of the positivity threshold in the PET processing methods section.
All participants were community-dwelling adults recruited locally from advertisements, public talks, and letters. Participants had a Mini-Mental State Examination score $\geq 26$ at baseline. Participants were excluded on the basis of screening for neurologic and psychiatric disorders, loss of consciousness $>10$ minutes, drug or alcohol abuse, major heart surgery, or chemotherapy within 5 years. All were fluent English speakers and right-handed.

\section{Standard protocol approvals, registrations, and patient consents}

This study was approved by the Institutional Review boards from the University of Texas Southwestern Medical Center and the University of Texas at Dallas. All participants provided written informed consent and were debriefed according to human investigations committee guidelines.

\section{Cognition}

From a larger cognitive battery, we focused on measures of episodic memory performance, including the following tasks: delayed recall and delayed recognition of the Hopkins Verbal Learning test ${ }^{12}$ and the immediate recall of the CANTAB Verbal Recognition Memory task. ${ }^{13}$ Baseline scores were converted to $z$ scores in the full sample, and the follow-up scores were $z$ transformed with the use of the baseline mean and standard deviation. The $z$ scores were averaged to form an episodic memory composite. Change was measured as the difference between baseline and follow-up. Additional analyses also included composites for processing speed (Wechsler Adult Intelligence Scale Digit Symbol, ${ }^{14}$ Digit Comparison $\left.{ }^{15,16}\right)$ and reasoning (Raven's Progressive Matrices, ${ }^{17}$ Educational Testing Service Letter Sets ${ }^{18}$ ).

\section{MRI protocol}

Participants were scanned on the same 3T Philips Achieva (Philips, Best, the Netherlands) scanner with an 8-channel head coil at baseline and follow-up. High-resolution anatomic images were collected with a T1-weighted magnetizationprepared rapid-acquisition gradient echo sequence with 160 sagittal slices, a field of view of $204 \times 256 \times 160 \mathrm{~mm}$, voxel size of $1 \times 1 \times 1 \mathrm{~mm}^{3}$, repetition time of 8.1 milliseconds, echo time of 3.7 milliseconds, and flip angle of $12^{\circ}$. Time 1 and 2 anatomic images were processed separately with the FreeSurfer version 5.3 cross-sectional pipeline (surfer.nmr.mgh. harvard.edu/ $/)^{19,20}$ with thorough manual editing, as detailed previously. ${ }^{21}$ Eight bilateral regions of interest (ROIs) were derived from FreeSurfer cortical parcellations with the Desikan-Killiany atlas, ${ }^{22}$ made up of most of the neocortex (excluding primary sensory and motor regions and the medial temporal lobe) and corresponding with previous amyloid imaging studies from this sample $e^{23-25}$ : posterior cingulate 
(PCC), anterior cingulate, lateral prefrontal (LPFC), orbitofrontal, precuneus, lateral parietal, lateral occipital, and lateral temporal cortices.

FreeSurfer parcellations were also used to compute 3 measures of brain structure: hippocampal volume, global cortical volume (sum of all cortical parcellations), and a composite of $\mathrm{AD}$ signature regions, ${ }^{26,27}$ including entorhinal, fusiform, parahippocampal, midtemporal, inferior-temporal, and inferior parietal cortices. Adjusted volumes were computed separately at each time point with the following equation: adjusted volume $=$ raw volume $-\mathrm{b} \times($ intracranial volume group mean intracranial volume), where $\mathrm{b}$ is the slope of regression of an ROI volume on intracranial volume. ${ }^{28}$ Atrophy (change in adjusted volume) was computed as a difference score.

\section{PET acquisition}

The PET protocol was the same at baseline and follow-up, following previously reported procedures. ${ }^{23}$ All participants were injected with a $370-\mathrm{MBq}(10 \mathrm{mCi})$ bolus of ${ }^{18} \mathrm{~F}$-florbetapir. ${ }^{3}$ At 30 minutes after injection, participants were positioned on the imaging table of a Siemens ECAT HR PET scanner (Siemens, Munich, Germany). A 2-minute scout was acquired to ensure that the participant's brain was completely in the field of view and that there was no rotation in either plane. A 2 frame by 5 minutes each dynamic emission acquisition was started 50 minutes after injection, and immediately after, an internal rod source transmission scan was acquired for 7 minutes. The transmission image was reconstructed using back-projection and a 6-mm full width at half-maximum gaussian filter. The emission images were processed by iterative reconstruction, with 4 iterations and 16 subsets and a 3-mm full width at half-maximum ramp filter.

\section{PET preprocessing}

To improve the measurement of change in amyloid over time, additional preprocessing steps were taken to minimize noise across time points. First, to avoid biasing the change measurement to either time point, each participant's baseline MRI and follow-up MRI were used to create the individual's mean MRI with FreeSurfer version 5.3. PET images from each time point were linearly coregistered to the MRI at the corresponding time point with FLIRT $^{29}$ and then transformed to mean MRI space with the transformation matrix derived from coregistration of the MRI at each time point to the mean MRI. Second, bilateral FreeSurfer ROIs from each time point were transformed to mean MRI space and thresholded at 0.7 , thus eroding ROI borders and reducing partial volume effects. ${ }^{30}$ Next, to generate a single set of ROI masks, ROIs from each time point were combined into a single conjunction mask that included only voxels present at both time points. These conjunction masks ensured that additional error would not arise from differences in parcellations across time, including differences due to atrophy. Finally, we used a reference region previously shown to provide the most stable reference over time: the average of both the whole cerebellum and the cerebral white matter. ${ }^{31}$ A partial volume correction with the method of Muller-Gartner et al. ${ }^{32}$ was tested but was found to add error, particularly due to limitations in coregistering PET to $M R{ }^{33}$ and therefore the data presented are not partial volume corrected.

SUVRs were computed for each conjunction mask ROI at each time point and the mean across the 8 ROIs (global SUVR). Change in SUVR in each ROI and global SUVR was computed as a difference score between baseline and follow-up.

All participants were classified as amyloid positive or negative at baseline on the basis of whether they fell above or below a study-specific cutoff (SUVR 0.76) of 2 SD above the mean global SUVR in young adults. ${ }^{23}$ This low threshold was selected to reduce the number of false negatives and to focus on individuals with initially very low global SUVRs.

\section{Statistical analysis}

Separate general linear models (GLMs) were used to assess whether there were significant changes (main effect of time) in amyloid and measures of brain structure across the full sample of initially amyloid-negative adults (age 30-89 years at baseline), as well as within the middle-aged subsample (age 30-59 years at baseline), controlling for age, sex, and APOE.

In the primary analyses, multiple GLMs were conducted across all amyloid-negative adults and within the middle-aged subsample to assess whether the change in SUVR predicted (1) the change in episodic memory, (2) the change in measures of brain structure, and (3) the change in episodic memory after controlling for measures of brain structure. All analyses controlled for baseline SUVR, age, sex, and APOE, as well as baseline measures of episodic memory (models 1 and 3 ) and brain structure (models 2 and 3). Analyses were conducted first with global SUVR change (with baseline global SUVR as a covariate) and second as 8 separate GLMs for each of the ROIs (with baseline regional SUVR as a covariate). One participant was missing follow-up cognitive data and was excluded from cognitive analyses. All analyses were conducted with SPSS version 24 (SPSS Inc, Chicago, IL).

\section{Data availability}

Baseline data are online (fcon_1000.projects.nitrc.org/indi/ retro/dlbs.html), and follow-up data are available to qualified investigators on request to the corresponding and senior authors.

\section{Results}

\section{Longitudinal changes in amyloid and brain structure in initially amyloid-negative adults}

Table 1 shows the sample characteristics for all initially amyloid-negative adults and the middle-aged subsample of 
Table 1 Sample characteristics

\begin{tabular}{|c|c|c|}
\hline & $\begin{array}{l}\text { Initially amyloid-negative adults } \\
\text { (age } 30-89 \mathrm{y}, \mathrm{n}=126 \text { ) }\end{array}$ & $\begin{array}{l}\text { Middle-aged subsample } \\
\text { (age } 30-59 \text { y, } n=48 \text { ) }\end{array}$ \\
\hline Age, y & $63.16(13.39)$ & $48.96(8.49)$ \\
\hline Time between cognitive sessions, y & $3.79(0.32)$ & $3.85(0.32)$ \\
\hline Time between PET scans, y & $3.61(0.41)$ & $3.57(0.39)$ \\
\hline Education, y & $15.40(2.14)$ & $15.39(1.99)$ \\
\hline$A P O E \varepsilon 4$ carriers, $\mathrm{n}(\%)^{\mathrm{a}}$ & $27(21.8)^{a}$ & $15(32.6)^{a}$ \\
\hline Female sex, $\mathrm{n}(\%)$ & $74(58.7)^{a}$ & $27(56.2)^{a}$ \\
\hline Black, n (\%) ${ }^{a}$ & $8(6.3)$ & $3(6.3)$ \\
\hline Baseline MMSE score & $28.48(1.18)$ & $28.48(1.31)$ \\
\hline MMSE score change & $0.52(1.56)$ & $0.56(1.62)$ \\
\hline Baseline episodic memory, z score & $0.03(0.86)$ & $0.28(0.93)$ \\
\hline Episodic memory change, z score & $-0.04(0.80)$ & $-0.09(0.83)$ \\
\hline Baseline reasoning, $z$ score & $0.06(0.88)$ & $0.42(0.84)$ \\
\hline Reasoning change, $z$ score & $-0.03(0.50)$ & $0.01(0.45)$ \\
\hline Baseline processing speed, $z$ score & $0.08(0.95)$ & $0.66(0.93)$ \\
\hline Processing speed change, $z$ score & $-0.31(0.45)$ & $-0.23(0.51)$ \\
\hline Baseline global SUVR & $0.70(0.03)$ & $0.70(0.03)$ \\
\hline Global SUVR change & $0.009(0.030)$ & $0.002(0.023)$ \\
\hline Baseline global cortical volume, $\mathrm{mm}^{3} \times 100,000$ & $4.22(0.48)$ & $4.51(0.47)$ \\
\hline Global cortical volume change, $\mathrm{mm}^{3} \times 100,000$ & $-0.14(0.16)$ & $-0.10(0.13)$ \\
\hline Baseline hippocampal volume, $\mathrm{mm}^{3} \times 1,000$ & $7.97(0.9)$ & $8.50(0.68)$ \\
\hline Hippocampal volume change, $\mathrm{mm}^{3} \times 1,000$ & $-0.28(0.53)$ & $-0.17(0.43)$ \\
\hline Baseline AD signature volume, $\mathrm{mm}^{3} \times 1,000$ & $89.73(7.70)$ & $94.07(7.09)$ \\
\hline$A D$ signature volume change, $\mathrm{mm}^{3} \times 1,000$ & $-2.90(3.61)$ & $-1.88(3.25)$ \\
\hline
\end{tabular}

Abbreviations: $A D$ = Alzheimer disease; $\mathrm{MMSE}=$ Mini-Mental State Examination; $\mathrm{SUVR}$ = standardized uptake value ratio.

Sample characteristics are shown for the full sample of all initially amyloid-negative adults (age 30-89 years, left column) and for the middle-aged subsample (age 30-59 years, right column).

a Continuous data are reported as mean (SD); categorical data (sex, $A P O E$, and ethnicity) are reported as number (percent).

adults 30 to 59 years of age. There was a significant mean increase over time in global amyloid burden $(F=3.876, p=$ $0.05)$, as well as significant decreases in hippocampal volume $(F=8.091, p=0.005), \mathrm{AD}$ signature region volume $(F=$ $12.581, p<0.001)$, and global cortical volume $(F=9.645, p=$ 0.002 ). When analyses were restricted only to the subsample of adults 30 to 59 years of age, there was not a significant mean change over time in global amyloid burden $(F=0.313, p=$ $0.577)$ or $\mathrm{AD}$ signature region volume $(F=1.99, p=0.162)$, but there were significant decreases in hippocampal volume $(F=10.278, p=0.002)$ and global cortical volume $(F=$ $11.342, p=0.001)$.

Figure 1 presents the individual changes both in global SUVR and within each of the 8 ROIs as a function of age. As expected, we observed both random fluctuations (both increases and decreases) likely due to noise and larger increases that likely reflect amyloid accumulation. As figure 1A shows, 14 of 126 initially amyloid-negative participants (11.1\%) crossed the threshold for positivity after the 4-year follow-up, only 1 of whom was $<60$ years of age (a 58-yearold). Converters were older $(t=2.813, p=0.006)$ and had greater baseline global SUVR $(t=3.893, p<0.001)$ than nonconverters but did not differ in terms of sex, APOE status, or any baseline or change measures of cortical volume or episodic memory $(p>0.15)$.

An ROI $\times$ time analysis on SUVR, with ROI as a withinparticipant variable, revealed that there were significant baseline differences in SUVR by region (main effect of ROI, 


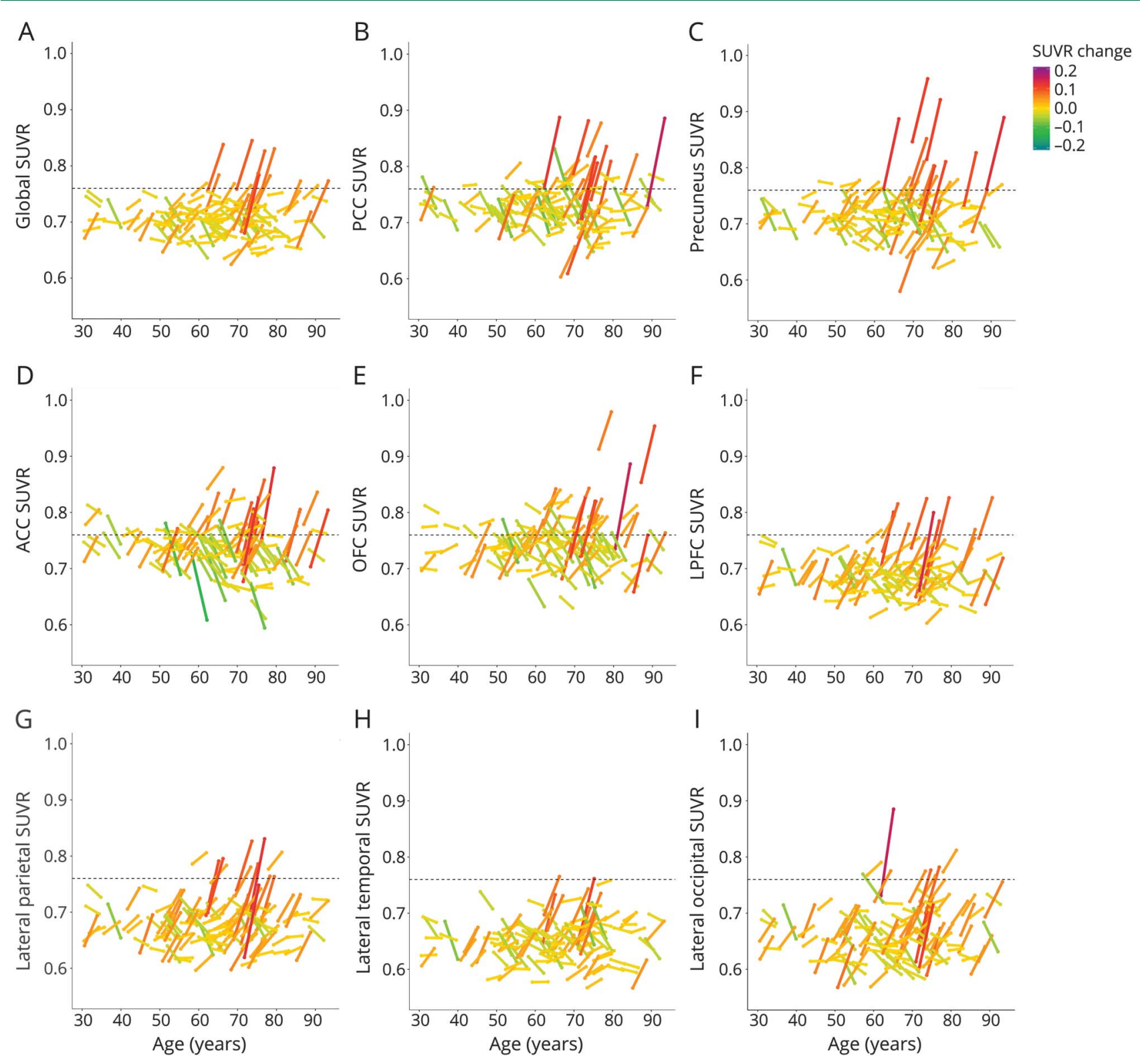

Lines represent each individual's raw change in standardized uptake value ratio (SUVR) for (A) global SUVR and (B-I) each of the 8 regions of interest (ROIs), color-coded by the magnitude of change. Large increases are shown in warmer (red) colors; no change is shown in yellow; and decreases are shown in cooler (green) colors. The dashed line on each figure represents the positivity threshold, set at an SUVR of 0.76 based on global SUVR. ACC = anterior cingulate cortex; LPFC = lateral prefrontal cortex; OFC = orbitofrontal cortex; PCC = posterior cingulate cortex.

$F=167.263, p<0.001)$. Higher baseline SUVR was present for PCC, precuneus, orbitofrontal cortex, and anterior cingulate cortex compared to lateral parietal, lateral temporal, and lateral occipital cortices (figure 1, B-I). The smallest difference between these 2 sets of ROIs (between the LPFC and precuneus) is significantly different $(t=-9.394, p<$ 0.001 ). Although overall SUVR significantly increased over time (main effect of time, $F=23.447, p<0.001$ ), the mean change in SUVR did not significantly differ across regions (ROI $\times$ time interaction, $F=1.017, p=0.417$ ). Nevertheless, regional differences may exist in the effect of SUVR change on cognitive decline.

\section{Amyloid accumulation and cognitive decline in initially amyloid-negative adults}

Using GLM, we assessed whether the global SUVR change score predicted the episodic memory change score, treating baseline global SUVR, baseline episodic memory performance, age, $A P O E$, and sex as covariates. Results are summarized in table 2. Increasing change in global SUVR marginally predicted declining episodic memory, while baseline global SUVR did not predict change in episodic memory. When restricted to only adults 30 to 59 years of age, increasing change in global SUVR was significantly associated with declining episodic memory, while baseline global SUVR again 
Table 2 Global and regional SUVR change on episodic memory change results

\begin{tabular}{|c|c|c|c|c|c|c|c|c|c|}
\hline & \multicolumn{9}{|l|}{ Region } \\
\hline & Global & PCC & Precuneus & $\begin{array}{l}\text { Lateral } \\
\text { parietal }\end{array}$ & $\begin{array}{l}\text { Lateral } \\
\text { occipital }\end{array}$ & $\begin{array}{l}\text { Lateral } \\
\text { temporal }\end{array}$ & OFC & LPFC & ACC \\
\hline \multicolumn{10}{|c|}{ Initially amyloid-negative adults } \\
\hline \multicolumn{10}{|c|}{ SUVR change } \\
\hline$F$ value & 3.820 & $6.284^{a}$ & $7.948^{\mathrm{a}}$ & $6.211^{\mathrm{a}}$ & 3.077 & 1.655 & 0.106 & 1.424 & 0.010 \\
\hline$p$ Value & 0.053 & $0.014^{\mathrm{a}}$ & $0.006^{\mathrm{a}}$ & $0.014^{\mathrm{a}}$ & 0.082 & 0.201 & 0.745 & 0.235 & 0.920 \\
\hline \multicolumn{10}{|c|}{ Baseline SUVR } \\
\hline$F$ value & 2.152 & 1.889 & 0.474 & 0.752 & 0.814 & 1.466 & 2.797 & 0.132 & 1.789 \\
\hline$p$ Value & 0.145 & 0.172 & 0.492 & 0.387 & 0.369 & 0.228 & 0.097 & 0.717 & 0.184 \\
\hline \multicolumn{10}{|c|}{ Baseline memory performance } \\
\hline$F$ value & 58.238 & 62.283 & 61.572 & 59.618 & 57.886 & 57.000 & 54.549 & 56.535 & 54.377 \\
\hline$p$ Value & $<0.001$ & $<0.001$ & $<0.001$ & $<0.001$ & $<0.001$ & $<0.001$ & $<0.001$ & $<0.001$ & $<0.001$ \\
\hline \multicolumn{10}{|l|}{ Age } \\
\hline$F$ value & 0.219 & 1.191 & 1.383 & 1.639 & 2.336 & 2.535 & 2.453 & 2.061 & 1.671 \\
\hline$p$ Value & 1.529 & 0.277 & 0.242 & 0.203 & 0.129 & 0.114 & 0.120 & 0.154 & 0.199 \\
\hline \multicolumn{10}{|l|}{ Sex } \\
\hline$F$ value & 2.414 & 1.894 & 2.503 & 2.182 & 2.125 & 2.730 & 2.476 & 1.991 & 1.742 \\
\hline$p$ Value & 0.123 & 0.171 & 0.116 & 0.142 & 0.148 & 0.101 & 0.118 & 0.161 & 0.190 \\
\hline \multicolumn{10}{|l|}{$A P O E$} \\
\hline$F$ value & 0.948 & 1.345 & 0.840 & 0.939 & 1.157 & 0.926 & 1.664 & 1.240 & 2.115 \\
\hline$p$ Value & 0.332 & 0.248 & 0.361 & 0.335 & 0.284 & 0.338 & 0.200 & 0.268 & 0.149 \\
\hline \multicolumn{10}{|c|}{30 - to $59-y$-olds } \\
\hline \multicolumn{10}{|c|}{ SUVR change } \\
\hline$F$ value & 6.927 & $8.613^{a}$ & $9.665^{\mathrm{a}}$ & $12.074^{a}$ & $16.077^{a}$ & 2.266 & 3.172 & 4.566 & 0.001 \\
\hline$p$ Value & 0.012 & $0.006^{a}$ & $0.004^{a}$ & $0.001^{a}$ & $<0.001^{a}$ & 0.141 & 0.083 & 0.039 & 0.971 \\
\hline \multicolumn{10}{|c|}{ Baseline SUVR } \\
\hline$F$ value & 2.381 & 1.251 & 1.049 & 4.667 & 7.016 & 1.536 & 0.014 & 3.113 & 1.203 \\
\hline$p$ Value & 0.131 & 0.270 & 0.312 & 0.037 & 0.012 & 0.223 & 0.905 & 0.086 & 0.280 \\
\hline \multicolumn{10}{|c|}{ Baseline memory performance } \\
\hline$F$ value & 18.212 & 17.676 & 21.985 & 20.963 & 27.604 & 17.766 & 14.410 & 16.289 & 14.711 \\
\hline$p$ Value & $<0.001$ & $<0.001$ & $<0.001$ & $<0.001$ & $<0.001$ & $<0.001$ & 0.001 & $<0.001$ & $<0.001$ \\
\hline \multicolumn{10}{|l|}{ Age } \\
\hline$F$ value & 0.007 & 0.227 & 0.066 & 0.020 & 0.325 & 0.021 & 0.000 & 0.045 & 0.044 \\
\hline$p$ Value & 0.934 & 0.636 & 0.799 & 0.889 & 0.572 & 0.885 & 0.997 & 0.833 & 0.835 \\
\hline \multicolumn{10}{|l|}{ Sex } \\
\hline$F$ value & 0.064 & 0.260 & 0.120 & 0.011 & 0.408 & 0.006 & 0.098 & 0.001 & 0.093 \\
\hline$p$ Value & 0.801 & 0.613 & 0.731 & 0.916 & 0.527 & 0.938 & 0.756 & 0.976 & 0.762 \\
\hline
\end{tabular}


Table 2 Global and regional SUVR change on episodic memory change results (continued)

\begin{tabular}{|c|c|c|c|c|c|c|c|c|c|}
\hline & \multicolumn{9}{|l|}{ Region } \\
\hline & Global & PCC & Precuneus & $\begin{array}{l}\text { Lateral } \\
\text { parietal }\end{array}$ & $\begin{array}{l}\text { Lateral } \\
\text { occipital }\end{array}$ & $\begin{array}{l}\text { Lateral } \\
\text { temporal }\end{array}$ & OFC & LPFC & ACC \\
\hline \multicolumn{10}{|l|}{$A P O E$} \\
\hline$F$ value & 1.925 & 1.487 & 3.402 & 3.159 & 3.088 & 1.984 & 1.636 & 1.776 & 2.080 \\
\hline$p$ Value & 0.174 & 0.230 & 0.073 & 0.084 & 0.087 & 0.167 & 0.209 & 0.191 & 0.158 \\
\hline \multicolumn{10}{|c|}{$\begin{array}{l}\text { Abbreviations: ACC = anterior cingulate cortex; LPFC = lateral prefrontal cortex; OFC = orbitofrontal cortex; PCC = posterior cingulate cortex; SUVR = } \\
\text { standardized uptake value ratio. } \\
\text { General linear model }(G L M) \text { results are shown for global SUVR change and for each of } 8 \text { regions of interest (ROIs) on episodic memory change for all initially } \\
\text { amyloid-negative adults and the middle-aged subsample. The following covariates are also reported: baseline SUVR, baseline memory, age, sex, and APOE. } \\
\text { For global SUVR and each ROI, the corresponding SUVR change and baseline SUVR measure were included in each respective GLM. } \\
\text { a } p<0.05 \text { after false discovery rate correction for multiple comparisons. }\end{array}$} \\
\hline
\end{tabular}

did not significantly predict memory decline. Together, these results indicate that change in global SUVR is more predictive than baseline SUVR of declining memory in initially amyloidnegative adults.

Next, we assessed whether specific regions were sensitive markers of an early amyloid-cognition relationship (table 2). In all initially amyloid-negative adults, increasing change in SUVR in the PCC, precuneus, and lateral parietal cortices predicted declining episodic memory (table 2) even after a false discovery rate correction for multiple comparisons. In adults 30 to 59 years of age, the relationship between regional SUVR change and episodic memory decline remained significant for PCC, precuneus, and lateral parietal cortices (table 2). In addition, SUVR change in the lateral occipital cortex and LPFC was associated with declining episodic memory in adults 30 to 59 years of age. The effect in the LPFC was mitigated to a trend after correction for multiple comparisons, but all other effects in the 30- to 59-year-old subsample survived.

Additional analyses in initially amyloid-negative adults (and the middle-aged subsample) were conducted for other nonmemory cognitive domains, but increasing SUVR was not significantly related to declines in processing speed or reasoning globally or to any ROIs $(p>0.10)$.

To further characterize the observed early amyloid-related changes in memory, a series of post hoc analyses were conducted. First, to assess whether these posterior cortical regions may provide a better target for detecting early amyloid-related changes in memory than global SUVR, a composite posterior cortical ROI (the mean of PCC, precuneus, lateral parietal, and lateral occipital cortices) was computed. In a model that included both global SUVR change and posterior cortical SUVR change, the posterior cortical ROI was significantly associated with declining memory $(F=$ 7.181, $p=0.008)$ in initially amyloid-negative adults, while global SUVR was not $(F=2.464, p=0.119)$. Likewise, in adults 30 to 59 years of age only, increasing change in SUVR in the posterior cortical ROI was again significantly associated with changing episodic memory $(F=8.351, p=0.006)$, while global SUVR change was only marginally significant $(F=$ 3.895, $p=0.056$ ).

In a second post hoc analysis, we assessed whether the relationship between changing amyloid and changing memory performance was apparent before classification as amyloid positive. Across all adults who were amyloid negative at follow-up, increasing amyloid in the PCC $(F=5.577, p=$ $0.020)$, precuneus $(F=7.238, p=0.008)$, and lateral parietal $(F=6.344, p=0.013)$ cortices remained significant predictors of declining episodic memory. When the analysis was restricted to only adults 30 to 59 years of age, the relationship between increasing amyloid in the PCC $(F=6.392, p=$ $0.016)$, precuneus $(F=9.088, p=0.005)$, lateral parietal $(F=$ $8.471, p=0.006)$, and lateral occipital $(F=10.719, p=0.002)$ cortices and declining episodic memory remained significant. Furthermore, those who converted to amyloid positive over the interval did not have greater memory decline than nonconverters $(F=0.183, p=0.669)$. Those who converted did not necessarily exhibit the largest increases in amyloid because some individuals who were just subthreshold at baseline crossed the threshold as a result of a very small increase, while others exhibited large increases but remained just below the positivity threshold at follow-up. Together, these findings suggest that it is the magnitude of change in amyloid rather than the crossing of a threshold that is most related to declining memory in initially amyloid-negative adults.

It is expected that SUVR change in the amyloid-negative sample necessarily includes random fluctuations in noise (both increases and decreases) and larger increases due to amyloid accumulation. To verify that the present findings were not driven by noise fluctuations, post hoc analyses were also conducted in 37 participants who exhibited SUVR increases above a test-retest cutoff of $2.5 \%$ change (equivalent to an SUVR change of 0.019 using the maximum baseline SUVR). ${ }^{34}$ In this smaller sample, the pattern of findings and the effect sizes remain similar to those of the full sample 
(figure 2), such that declining memory is associated with increasing SUVR change in the PCC $(r=-0.26, p=0.14)$ and precuneus $(r=-0.27, p=0.13)$, although the effects only approached statistical significance. Thus, while imposing a threshold results in reduced power, the similarity of the effect sizes provides some support that the observed effects in the larger amyloid-negative sample reflect the magnitude of the increase in amyloid rather than noise fluctuations.

\section{Amyloid accumulation and structural atrophy}

Next, we assessed whether increasing amyloid accumulation predicted increasing decline in hippocampal, global cortical, and $\mathrm{AD}$ signature region volume over the 4-year follow-up. Across all initially amyloid-negative adults, increasing global SUVR was marginally associated with declining hippocampal volume $(F=3.775, p=0.054)$ and global cortical volume $(F=3.020, p=0.085)$ (figure 3$)$ but not decline in $\mathrm{AD}$ signature region volume $(F=0.927, p=0.338)$. In adults 30 to 59 years of age, increasing global SUVR was significantly associated with declining hippocampal volume $(F=4.774, p$ $=0.035)$ but not with declining global cortical volume $(F=$ $0.000, p=0.998)$ or $\mathrm{AD}$ signature region volume $(F=0.094$, $p=0.757)$. When regional measures of SUVR were used, the relationship between SUVR change and volume change was not significant for any region after correction for multiple comparisons.

\section{Amyloid accumulation and cognitive decline after controlling for structural atrophy}

Finally, we assessed whether structural atrophy measures might modify the relationship between increasing regional SUVR and declining memory. All of the previously detected relationships between regional SUVR change and episodic memory decline remained significant after controlling for baseline and change in hippocampal volume and global cortical volume. Across all initially amyloid-negative adults, increasing change in SUVR in the PCC, precuneus, and lateral parietal cortices remained significantly associated with declining episodic memory (table 3). In adults 30 to 59 years of age only, the relationship between increasing change in SUVR in the PCC, precuneus, lateral parietal cortex, lateral occipital cortex, and LPFC $(F=5.711, p=$ 0.023 ) and declining episodic memory remained significant, and global SUVR was mitigated to a trend (table 3). None of the measures of atrophy significantly predicted declining episodic memory in any of these models, although baseline hippocampal volume was marginally associated with declining memory in the middle-aged subsample. Similarly, controlling for baseline volume and change in $\mathrm{AD}$ signature regions resulted in persisting significant effects of increasing change in SUVR in the PCC $(F=5.847, p=0.017)$, precuneus $(F=6.265$, $p=0.014)$, and lateral parietal $(F=4.260, p=0.041)$ cortices across all initially amyloid-negative participants and additional effects for global SUVR $(F=6.796, p=0.013)$ and lateral occipital $(F=16.032, p<0.001)$ for middle-aged participants.

Figure 2 Increasing regional amyloid accumulation in posterior cortical ROIs predicts declining episodic memory over 4 years
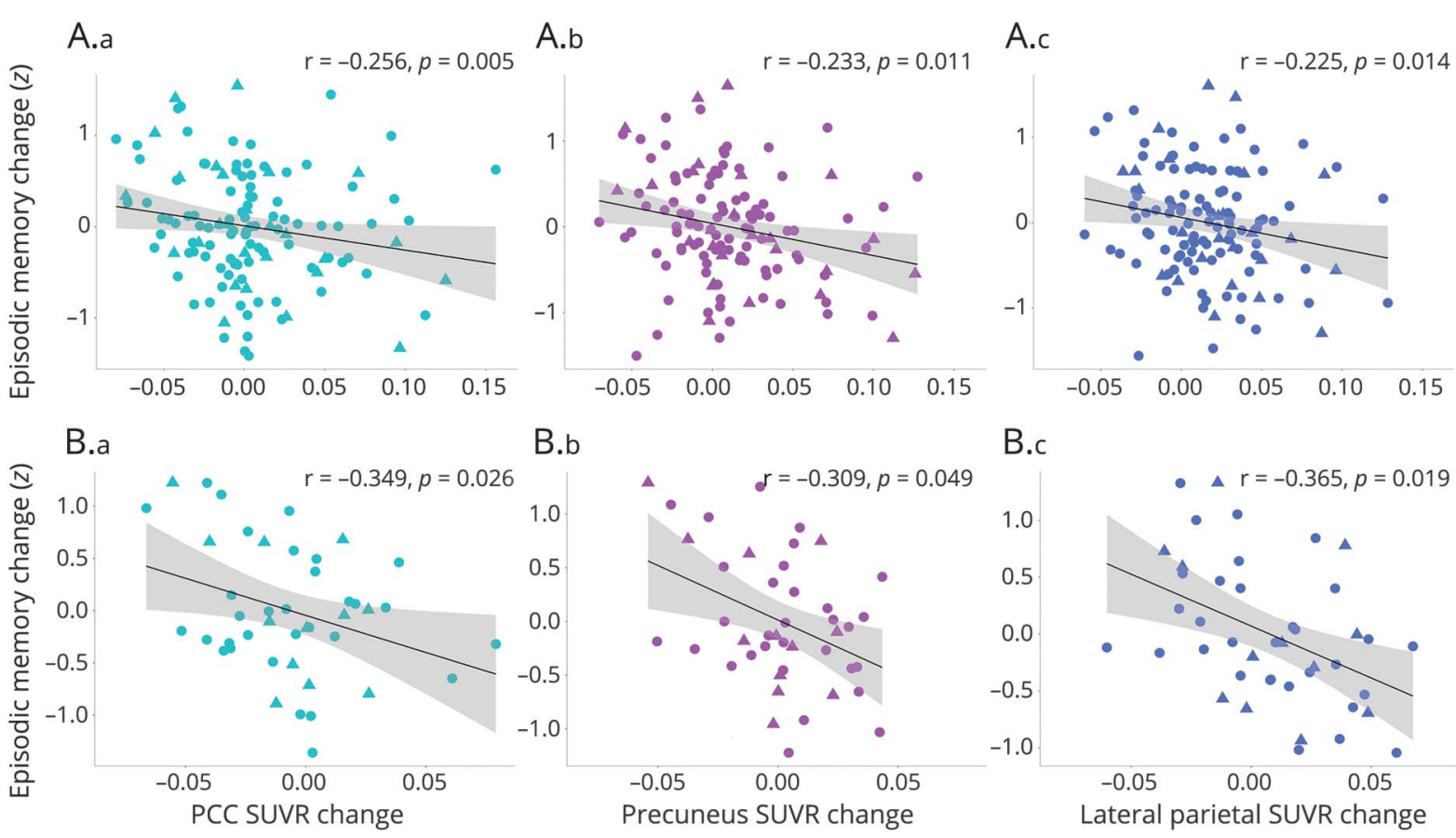

The correlations between standardized uptake value ratio (SUVR) change in each of the 3 posterior cortical regions of interest (ROI) (posterior cingulate [PCC], precuneus, lateral parietal cortices) and change in episodic memory are shown (A.a-A.c) for all initially amyloid-negative adults 30 to 89 years of age and (B.a-B.c) within the middle-aged subsample 30 to 59 years of age. Episodic memory change is measured as a residual after controlling for baseline memory and SUVR, as well as age, sex, and APOE. APOE status is coded by shape for descriptive purposes, with $\varepsilon 4$ carriers as triangles and noncarriers as circles. 

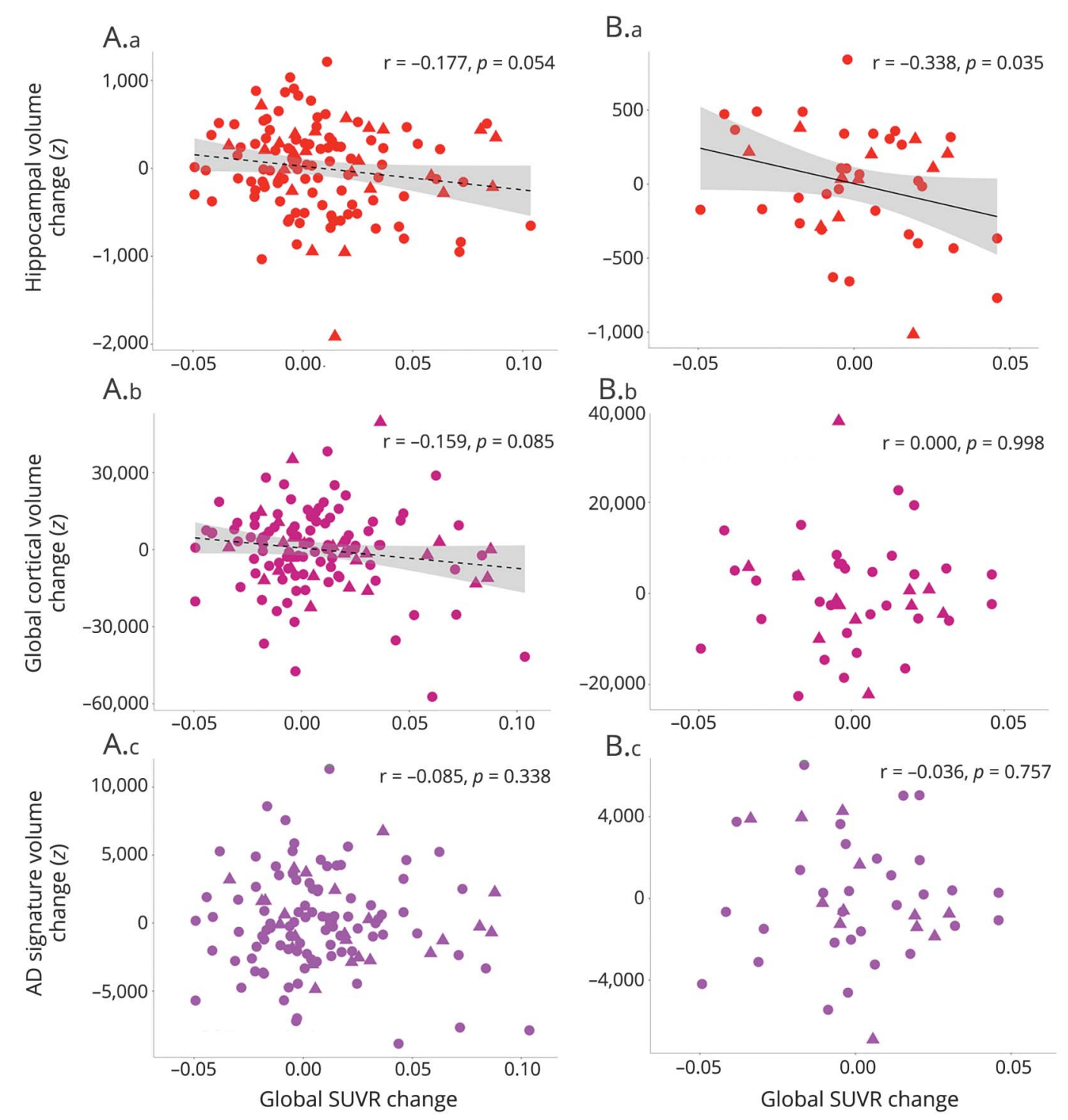

(A.a-A.c) In initially amyloid-negative adults 30 to 89 years of age, increasing global standardized uptake value ratio (SUVR) change is marginally related to (A.a) declining hippocampal volume and (A.b) global cortical volume but not (A.C) Alzheimer disease (AD) signature volume. (B.a-B.c) In the middle-aged subsample 30 to 59 years of age, increasing global SUVR was significantly related to (B.a) declining hippocampal volume but not (B.b) global cortical volume or (B.C) AD signature volume. Structural data are standardized residuals after controlling for baseline SUVR and the baseline measure of neurodegeneration, as well as age, sex, and APOE. APOE status is coded by shape for descriptive purposes, with $\varepsilon 4$ carriers as triangles and noncarriers as circles.

\section{Discussion}

Using initially amyloid-negative adults, the present study provides evidence of an early subclinical relationship between accumulating amyloid deposition over 4 years and concurrent declines in episodic memory. Notably, posterior cortical regions drove the relationship between accumulating amyloid and declining memory, suggesting that these regions may be useful in monitoring the progression of amyloid pathology and its relationship to memory decline from a very early stage. Furthermore, the relationship between amyloid and memory was evident starting in middle age, emphasizing the need for intervention early in the lifespan. Finally, these early amyloidrelated changes in episodic memory persisted after controlling for hippocampal atrophy and broader changes in $\mathrm{AD}$ signature regions and global cortical volume.

Our results corroborate recent findings that demonstrated a relationship between accumulating amyloid and declining memory in initially amyloid-negative adults using data from the Alzheimer's Disease Neuroimaging Initiative. ${ }^{4}$ Together, these 2 independent samples indicate that there are subtle but reliable amyloid-related declines in memory below traditional thresholds of positivity. Notably, the present study found that it is change in amyloid rather than baseline amyloid burden that is a sensitive marker of memory decline at this early stage. In contrast, studies in amyloid-positive adults have found that baseline amyloid was a better predictor of cognitive change 
Table 3 Global and posterior ROI SUVR change on episodic memory change results after controlling for measures of structural atrophy

\begin{tabular}{|c|c|c|c|c|c|c|c|c|c|c|}
\hline & \multicolumn{10}{|l|}{ Region } \\
\hline & \multicolumn{2}{|l|}{ Global } & \multicolumn{2}{|l|}{ PCC } & \multicolumn{2}{|c|}{ Precuneus } & \multicolumn{2}{|c|}{ Lateral parietal } & \multicolumn{2}{|c|}{ Lateral occipital } \\
\hline & $F$ value & $p$ Value & $F$ value & $p$ Value & $F$ value & $p$ Value & $F$ value & $p$ Value & $F$ value & $p$ Value \\
\hline \multicolumn{11}{|l|}{$\begin{array}{l}\text { Initially amyloid- } \\
\text { negative adults }\end{array}$} \\
\hline SUVR change & 3.537 & 0.063 & 6.503 & 0.012 & 7.278 & 0.008 & 5.677 & 0.019 & 3.523 & 0.063 \\
\hline Baseline SUVR & 1.632 & 0.204 & 1.807 & 0.182 & 0.342 & 0.56 & 0.546 & 0.462 & 0.685 & 0.41 \\
\hline Baseline memory & 56.318 & $<0.001$ & 60.238 & $<0.001$ & 59.114 & $<0.001$ & 57.743 & $<0.001$ & 56.893 & $<0.001$ \\
\hline $\begin{array}{l}\text { Baseline global } \\
\text { cortical volume }\end{array}$ & 0.201 & 0.655 & 0 & 0.998 & 0.157 & 0.693 & 0.2 & 0.656 & 0.348 & 0.557 \\
\hline $\begin{array}{l}\text { Global cortical } \\
\text { volume change }\end{array}$ & 1.274 & 0.261 & 1.291 & 0.258 & 1.371 & 0.244 & 1.4 & 0.239 & 2.527 & 0.115 \\
\hline $\begin{array}{l}\text { Baseline } \\
\text { hippocampal volume }\end{array}$ & 0.142 & 0.707 & 0.022 & 0.882 & 0.003 & 0.959 & 0.191 & 0.663 & 0.287 & 0.593 \\
\hline Hippocampal change & 0.963 & 0.328 & 1.514 & 0.221 & 1.05 & 0.308 & 0.882 & 0.35 & 1.006 & 0.318 \\
\hline Age & 1.013 & 0.316 & 1.019 & 0.315 & 0.625 & 0.431 & 1.13 & 0.29 & 1.418 & 0.236 \\
\hline Sex & 2.365 & 0.127 & 1.308 & 0.255 & 2.315 & 0.131 & 2.192 & 0.142 & 2.497 & 0.117 \\
\hline APOE & 1.666 & 0.199 & 2.155 & 0.145 & 1.481 & 0.226 & 1.677 & 0.198 & 2.094 & 0.151 \\
\hline \multicolumn{11}{|l|}{ 30- to 59-y-olds } \\
\hline SUVR change & 3.682 & 0.064 & 8.993 & 0.005 & 8.468 & 0.006 & 11.05 & 0.002 & 14.144 & 0.001 \\
\hline Baseline SUVR & 9.358 & 0.004 & 1.899 & 0.178 & 0.802 & 0.377 & 4.593 & 0.04 & 8.729 & 0.006 \\
\hline Baseline memory & 20.627 & $<0.001$ & 18.528 & $<0.001$ & 21.935 & $<0.001$ & 21.98 & $<0.001$ & 30.176 & $<0.001$ \\
\hline $\begin{array}{l}\text { Baseline global } \\
\text { cortical volume }\end{array}$ & 1.127 & 0.296 & 0.873 & 0.357 & 0.868 & 0.358 & 0.3 & 0.588 & 0.064 & 0.803 \\
\hline $\begin{array}{l}\text { Global cortical } \\
\text { volume change }\end{array}$ & 4.072 & 0.052 & 2.103 & 0.156 & 1.99 & 0.168 & 1.794 & 0.19 & 1.88 & 0.18 \\
\hline $\begin{array}{l}\text { Baseline } \\
\text { hippocampal volume }\end{array}$ & 3.953 & 0.055 & 3.488 & 0.071 & 2.252 & 0.143 & 3.358 & 0.076 & 3.759 & 0.061 \\
\hline Hippocampal change & 0.349 & 0.559 & 0.249 & 0.621 & 0.007 & 0.934 & 0.118 & 0.734 & 0.312 & 0.58 \\
\hline Age & 0.25 & 0.621 & 0.01 & 0.921 & 0.006 & 0.937 & 0.302 & 0.586 & 1.169 & 0.288 \\
\hline Sex & 0.59 & 0.448 & 0.131 & 0.72 & 0.24 & 0.628 & 0.318 & 0.576 & 0.79 & 0.38 \\
\hline APOE & 3.431 & 0.073 & 2.522 & 0.122 & 4.035 & 0.053 & 3.774 & 0.061 & 3.777 & 0.061 \\
\hline
\end{tabular}

Abbreviations: $\mathrm{PCC}=$ posterior cingulate cortex; $\mathrm{ROI}=$ region of interest; SUVR = standardized uptake value ratio.

GLM results are shown for the effects of changing SUVR on changing memory after controlling for measures of structural atrophy, global SUVR change, and the 4 posterior cortical ROIs previously demonstrated to predict declining memory. Results are reported for all initially amyloid-negative adults (top) and the middle-aged subsample alone (bottom). The following covariates are also reported: baseline structural measures (hippocampal volume, global cortical volume), change in structural measures (hippocampal volume change, global cortical volume change), baseline SUVR, baseline memory, age, sex, and APOE. For global SUVR and each ROI, the corresponding SUVR change and baseline SUVR measure were included in each respective GLM.

than change in amyloid. ${ }^{35}$ This discrepancy likely results from differences in the utility of baseline SUVR as a meaningful measure of amyloid burden in amyloid-negative vs -positive adults. Greater baseline SUVR within the amyloid-negative range may reflect low but subthreshold amounts of amyloid, but it may also result from high nonspecific binding. As a result of the continuous and increasing nature of amyloid deposition in cognitively normal adults, ${ }^{9}$ longitudinal measurement of
SUVR change provides a more meaningful measurement of amyloid in individuals below the positivity threshold, which in turn enables detection of early amyloid-related changes in episodic memory.

It is also notable that amyloid-related cognitive changes were detected for memory but not for the other tested cognitive domains, including processing speed and reasoning, consistent 
with previous evidence of pivotal role of memory decline early in $\mathrm{AD}$ pathologic progression. ${ }^{4,23,36,37}$

The present study also yielded findings in a rarely studied group: middle-aged adults. Our results provide evidence that middle-aged adults may be an optimal target for early intervention because subtle but detectable changes in amyloid are already related to declining memory at this early stage in the lifespan. The associations between increasing amyloid and both declining memory and hippocampal volume were stronger in middle-aged than in older initially amyloidnegative adults. It may be that the detection of amyloid effects is more robust in middle age due to fewer confounds with other age-related diseases that may obscure the effects of amyloid later in life. We note that we did not find an effect of APOE \&4 despite previous evidence of its salience in middle age, ${ }^{38}$ but a larger sample of $\varepsilon 4$ carriers is likely needed to examine the genetic component.

We also found that the relationship between amyloid accumulation and memory decline persisted after the exclusion of converters to positivity and that memory did not decline significantly on the basis of only conversion to positivity. These findings suggest that it is the magnitude of change in amyloid rather than the crossing of an artificial threshold that is most related to declining memory in initially amyloidnegative adults. Furthermore, in combination with the findings in middle-aged participants and previous findings from other studies, ${ }^{4,39}$ these results emphasize that if the success of antiamyloid therapies requires intervention before downstream effects of amyloid on cognition, waiting until individuals are older and amyloid positive may be too late. The relationship between increasing amyloid accumulation and declining memory was localized to posterior cortical regions, including the PCC, precuneus, parietal, and occipital cortices. This effect was most robust in the PCC, which is often implicated as one of the earliest sites of amyloid accumulation. 8 These findings suggest that focusing on posterior cortical regions, particularly the posterior cingulate, may improve the ability to detect and monitor early amyloid-related changes in memory.

Further work is needed to clarify the mechanism underlying this early regionally specific effect of amyloid on memory. The present findings suggest that early amyloid-related memory decline may occur independently of morphometric changes in hippocampal, $\mathrm{AD}$ signature, or global cortical volume. While the lack of evidence that structural atrophy in these regions modulates the amyloid-memory decline relationship may reflect insufficient power or biased dropout, it is alternatively likely that the coarse measures of volumetric change used in the present study may not sufficiently capture the subtle neurodegenerative changes underlying amyloid-related memory decline at this early stage. In vitro and rat studies have demonstrated that amyloid results in synaptic dysfunction, ${ }^{40-42}$ and PET studies have demonstrated that amyloid is associated with disrupted functional connectivity in posterior networks important for memory. ${ }^{43-45}$ Thus, the observed amyloid-related memory decline in amyloidnegative adults may reflect local neurodegenerative effects of amyloid on synaptic dysfunction, and future research using fMRI and fluorodeoxyglucose may help to examine this possibility. Alternatively, these regional effects may be mediated by tau pathology in functionally and structurally connected mediotemporal lobe regions and its neurodegenerative effects. ${ }^{46}$ Notably, a recent study ${ }^{39}$ demonstrated in initially amyloid-negative adults that longitudinal increases in amyloid are associated with increased tau pathology. Future research incorporating tau and other neurodegenerative markers may therefore help to clarify the mechanism underlying these early effects of amyloid on memory decline and to elucidate the early pathologic progression of $\mathrm{AD}$.

An important limitation of the present study and all research focusing on amyloid-negative adults is the uncertainty that SUVR in this lower range reflects amyloid rather than sources of noise such as nonspecific binding and blood flow. Longitudinal measurement helps to identify individuals within the amyloid-negative range that harbor amyloid because amyloid should continue increasing. However, it is important to note that variability in change in noise is also expected on the basis of test-retest studies that have shown short-term noise fluctuations in SUVR of $2.5 \% .^{34}$ As a result of this test-retest variability, both increases and decreases in SUVR were observed, and it is presumed that larger increases, especially those above test-retest, are more likely to reflect changes in amyloid rather than noise. It is notable that individuals exhibiting SUVR decreases and increasing memory contribute to the primary finding of a relationship between increasing posterior cortical SUVR change and declining memory (figure 2). However, when we selectively analyzed participants with large increases above test-retest, we found a similar pattern of findings, although we lacked the power to detect statistical significance in this smaller sample. However, this finding provides some support that the direction and magnitude of the effect of SUVR change on episodic memory change reflect increases in amyloid rather than being driven by decreases in noise. Adding further credibility is the finding that the association between SUVR change and memory change is not explained by fluctuations in SUVR due to atrophy. Nevertheless, more research is needed to better elucidate the sources of noise and to understand the relative contributions of noise and amyloid deposition to the signal in the amyloidnegative range. Notably, these reliability issues are partially driven by having only 2 time points because of the longer follow-up interval in our aging study. Future research with additional time points will help to improve measurement reliability and accuracy.

It is also possible that the observed relationship was driven by memory decline, such that declining memory performance led to increased deposition of amyloid rather than amyloid leading to cognitive change. There is some evidence suggesting that poor neural efficiency (which may result in lower 
cognitive performance) may lead to greater amyloid deposition. ${ }^{47}$ However, multiple studies ${ }^{23,35,37,48}$ have demonstrated temporal precedence of amyloid over cognitive decline, providing support for the hypothesis that amyloid (directly or indirectly) leads to cognitive decline.

It is also notable that while subtle amyloid-related declines in episodic memory were observed, these participants remain cognitively normal. Continued follow-up is needed to assess whether those exhibiting increasing amyloid burden and declining memory will progress to dementia in the future. It should also be noted that $38 \%$ of the baseline sample was lost to follow-up 4 years later, although those who were retained did not significantly differ from those who failed to return. Slightly higher attrition rates were observed in the oldest participants (age 80-89 years) due to health problems. Biased dropout may therefore result in underestimation of effects in the population and in particular may contribute to our lack of findings regarding structural atrophy.

The present study demonstrates an early, regionally specific relationship between amyloid accumulation in posterior cortical regions and declining episodic memory in initially amyloid-negative adults. These findings highlight the importance of assessing regional changes in amyloid to monitor disease progression as early as middle age. Furthermore, our results suggest that early amyloid-related memory decline may be independent of hippocampal, $\mathrm{AD}$ signature region, and global cortical atrophy, although further research is needed to achieve greater clarity on the early pathologic sequence in $\mathrm{AD}$. Our findings align with other recent work ${ }^{4,39}$ to stress the importance of studying initially amyloid-negative adults to aid in the identification of a critical window for intervention before downstream effects of amyloid on neurodegeneration and cognitive decline occur.

\section{Author contributions}

Dr. Farrell designed the study, conducted the data analysis and interpretation, and wrote the paper. $\mathrm{Xi}$ Chen assisted with data processing and provided edits. Dr. Rundle provided administrative oversight of the study and edited the paper. Dr. Chan assisted with data processing and provided edits. Dr. Wig designed and oversaw the FreeSurfer protocol and provided edits to the paper. Dr. Park is the study principal investigator, contributed to the design of the study and data interpretation, and provided edits to the paper.

\section{Acknowledgment}

The authors thank Michael D. Rugg, PhD, Kristen M. Kennedy, $\mathrm{PhD}$, and Karen M. Rodrigue, $\mathrm{PhD}$ (all affiliated with University of Texas at Dallas), for advice and guidance on the current project. Additional thanks go to Patrick Evans, BS, Allison Parker, BS, Victor Faner, BS, Kristen Smart, BS, and Ashley Kuvet, BS, for data collection (all affiliated with University of Texas at Dallas). Thanks also go to Neil Savalia, BS, and Phillip Agres, MSc (University of Texas at Dallas), for FreeSurfer data processing and quality control.

\section{Study funding}

This study was supported by funding from the National Institute on Aging 5R37AG-006265 (D.P.) and RC1AG036199 (D.P.), as well as the Alzheimer's Association. The tracer was provided free of cost by Avid Radiopharmaceuticals, a wholly owned subsidiary of Eli Lilly, Inc, which also provided some funds for PET imaging.

\section{Disclosure}

The authors report no disclosures relevant to the manuscript. Go to Neurology.org/N for full disclosures.

\section{Publication history}

Received by Neurology March 5, 2018. Accepted in final form July 31, 2018.

\section{References}

1. Sperling R, Mormino E, Johnson K. The evolution of preclinical Alzheimer's disease: implications for prevention trials. Neuron 2014;84:608-622.

2. Klunk WE, Engler H, Nordberg A, et al. Imaging brain amyloid in Alzheimer's disease with Pittsburgh compound-B. Ann Neurol 2004;55:306-319.

3. Wong DF, Rosenberg PB, Zhou Y, et al. In vivo imaging of amyloid deposition in Alzheimer disease using the radioligand 18F-AV-45 (florbetapir [corrected] F 18). J Nucl Med 2010;51:913-920.

4. Landau SM, Horng A, Jagust WJ; Alzheimer's Disease Neuroimaging Initiative.Memory decline accompanies subthreshold amyloid accumulation. Neurology 2018;90:e1452-e1460.

5. Braak H, Braak E. Neuropathological stageing of Alzheimer-related changes. Acta Neuropathol 1991;82:239-259.

6. Sepulcre J, Sabuncu MR, Becker A, Sperling R, Johnson KA. In vivo characterization of the early states of the amyloid-beta network. Brain 2013;136:2239-2252.

7. Villain N, Chetelat G, Grassiot B, et al. Regional dynamics of amyloid-beta deposition in healthy elderly, mild cognitive impairment and Alzheimer's disease: a voxelwise PiB-PET longitudinal study. Brain 2012;135:2126-2139.

8. Grothe MJ, Barthel $\mathrm{H}$, Sepulcre J, et al. In vivo staging of regional amyloid deposition. Neurology 2017;89:2031-2038.

9. Palmqvist $\mathrm{S}$, Scholl $\mathrm{M}$, Strandberg $\mathrm{O}$, et al. Earliest accumulation of beta-amyloid occurs within the default-mode network and concurrently affects brain connectivity. Nat Commun 2017;8:1214.

10. Braak H, Thal DR, Ghebremedhin E, Del Tredici K. Stages of the pathologic process in Alzheimer disease: age categories from 1 to 100 years. J Neuropathol Exp Neurol 2011;70:960-969.

11. Bilgel M, An $Y$, Zhou $Y$, et al. Individual estimates of age at detectable amyloid onset for risk factor assessment. Alzheimers Dement 2016;12:373-379.

12. Brandt J. The Hopkins Verbal Learning Test: development of a new memory test with six equivalent forms. Clin Neuropsychol 1991;5:125-142.

13. Robbins TW, James M, Owen AM, Sahakian BJ, McInnes L, Rabbitt P. Cambridge Neuropsychological Test Automated Battery (CANTAB): a factor analytic study of a large sample of normal elderly volunteers. Dementia 1994;5:266-281.

14. Wechlser D. WMS-R Wechsler Memory Scale-Revised Manual. San Antonio: Psychological Corporation, Harcourt Brace Jovanovich, Inc; 1987.

15. Salthouse TA, Babcock RL. Decomposing adult age differences in working memory. Develop Psychol 1991;27:763-776.

16. Hedden T, Park DC, Nisbett R, Ji LJ, Jing Q, Jiao S. Cultural variation in verbal versus spatial neuropsychological function across the life span. Neuropsychology 2002;16:65-73.

17. Raven JC. Standard Progressive Matrices: Sets A, B, C, D \& E. Oxford: Oxford Psychologists Press; 1996.

18. Ekstrom RB, Harman HH. Manual for Kit of Factor-Referenced Cognitive Tests, 1976. Princeton: Educational Testing Service; 1976.

19. Dale AM, Fischl B, Sereno MI. Cortical surface-based analysis, I: segmentation and surface reconstruction. Neuroimage 1999;9:179-194.

20. Fischl B, Sereno MI, Dale AM. Cortical surface-based analysis, II: inflation, flattening, and a surface-based coordinate system. Neuroimage 1999;9:195-207.

21. Savalia NK, Agres PF, Chan MY, Feczko EJ, Kennedy KM, Wig GS. Motion-related artifacts in structural brain images revealed with independent estimates of in-scanner head motion. Hum Brain Mapp 2017;38:472-492.

22. Desikan RS, Segonne F, Fischl B, et al. An automated labeling system for subdividing the human cerebral cortex on MRI scans into gyral based regions of interest. Neuroimage 2006;31:968-980.

23. Farrell ME, Kennedy KM, Rodrigue KM, et al. Association of longitudinal cognitive decline with amyloid burden in middle-aged and older adults: evidence for a doseresponse relationship. JAMA Neurol 2017;74:830-838.

24. Kennedy KM, Rodrigue KM, Devous MD, et al. Effects of beta-amyloid accumulation on neural function during encoding across the adult lifespan. Neuroimage 2012;62:1-8

25. Rodrigue KM, Kennedy KM, Devous MD Sr, et al. Beta-amyloid burden in healthy aging: regional distribution and cognitive consequences. Neurology 2012;78:387-395. 
Dickerson BC, Bakkour A, Salat DH, et al. The cortical signature of Alzheimer's disease: regionally specific cortical thinning relates to symptom severity in very mild to mild $\mathrm{AD}$ dementia and is detectable in asymptomatic amyloid-positive individuals. Cereb Cortex 2009; 19:497-510.

27. Schwarz CG, Gunter JL, Wiste HJ, et al. A large-scale comparison of cortical thickness and volume methods for measuring Alzheimer's disease severity. Neuroimage Clin 2016;11:802-812.

28. Raz N, Lindenberger $\mathrm{U}$, Rodrigue $\mathrm{KM}$, et al. Regional brain changes in aging healthy adults: general trends, individual differences and modifiers. Cereb Cortex 2005;15:1676-1689.

29. Jenkinson M, Smith S. A global optimisation method for robust affine registration of brain images. Med Image Anal 2001;5:143-156.

30. Landau SM, Fero A, Baker SL, et al. Measurement of longitudinal beta-amyloid change with $18 \mathrm{~F}$-florbetapir PET and standardized uptake value ratios. J Nucl Med 2015;56:567-574.

31. Schwarz CG, Senjem ML, Gunter JL, et al. Optimizing PiB-PET SUVR change-overtime measurement by a large-scale analysis of longitudinal reliability, plausibility, separability, and correlation with MMSE. Neuroimage 2017;144:113-127.

32. Muller-Gartner HW, Links JM, Prince JL, et al. Measurement of radiotracer concentration in brain gray matter using positron emission tomography: MRI-based correction for partial volume effects. J Cereb Blood Flow Metab 1992;12:571-583.

33. Schwarz CG, Jones DT, Gunter JL, et al. Contributions of imprecision in PET-MRI rigid registration to imprecision in amyloid PET SUVR measurements. Hum Brain Mapp 2017.

34. Joshi AD, Pontecorvo MJ, Clark CM, et al. Performance characteristics of amyloid PET with florbetapir F 18 in patients with Alzheimer's disease and cognitively normal subjects. J Nucl Med 2012;53:378-384.

35. Villemagne VL, Burnham S, Bourgeat P, et al. Amyloid beta deposition, neurodegeneration, and cognitive decline in sporadic Alzheimer's disease: a prospective cohort study. Lancet Neurol 2013;12:357-367.

36. Sperling RA, Aisen PS, Beckett LA, et al. Toward defining the preclinical stages of Alzheimer's disease: recommendations from the National Institute on Aging-Alzheimer's Association workgroups on diagnostic guidelines for Alzheimer's disease. Alzheimers Dement 2011;7:280-292.
37. Lim YY, Maruff P, Pietrzak RH, et al. Effect of amyloid on memory and non-memory decline from preclinical to clinical Alzheimer's disease. Brain 2014;137:221-231.

38. Corder EH, Saunders AM, Strittmatter WJ, et al. Gene dose of apolipoprotein E type 4 allele and the risk of Alzheimer's disease in late onset families. Science 1993;261. 921-923.

39. Leal SL, Lockhart SN, Maass A, Bell RK, Jagust WJ. Subthreshold amyloid predicts tau deposition in aging. J Neurosci 2018;38:4482-4489.

40. Cleary JP, Walsh DM, Hofmeister JJ, et al. Natural oligomers of the amyloid-beta protein specifically disrupt cognitive function. Nat Neurosci 2005;8:79-84.

41. Shankar GM, Bloodgood BL, Townsend M, Walsh DM, Selkoe DJ, Sabatini BL. Natural oligomers of the Alzheimer amyloid-beta protein induce reversible synapse loss by modulating an NMDA-type glutamate receptor-dependent signaling pathway. J Neurosci 2007;27:2866-2875.

42. Walsh DM, Klyubin I, Fadeeva JV, Rowan MJ, Selkoe DJ. Amyloid-beta oligomers: their production, toxicity and therapeutic inhibition. Biochem Soc Trans 2002;30 552-557.

43. Hedden T, Van Dijk KR, Becker JA, et al. Disruption of functional connectivity in clinically normal older adults harboring amyloid burden. J Neurosci 2009;29: 12686-12694.

44. Sheline YI, Morris JC, Snyder AZ, et al. APOE4 allele disrupts resting state fMRI connectivity in the absence of amyloid plaques or decreased CSF Abeta42. J Neurosci 2010;30:17035-17040.

45. Sperling RA, Laviolette PS, O'Keefe K, et al. Amyloid deposition is associated with impaired default network function in older persons without dementia. Neuron 2009; 63:178-188.

46. Jacobs HIL, Hedden T, Schultz AP, et al. Structural tract alterations predict downstream tau accumulation in amyloid-positive older individuals. Nat Neurosci 2018;21: $424-431$.

47. Jagust WJ, Mormino EC. Lifespan brain activity, beta-amyloid, and Alzheimer's disease. Trends Cogn Sci 2011;15:520-526.

48. Petersen RC, Wiste HJ, Weigand SD, et al. Association of elevated amyloid levels with cognition and biomarkers in cognitively normal people from the community. JAMA Neurol 2016;73:85-92. 


\section{Neurology}

\section{Regional amyloid accumulation and cognitive decline in initially amyloid-negative adults}

Michelle E. Farrell, Xi Chen, Melissa M. Rundle, et al.

Neurology 2018;91;e1809-e1821 Published Online before print October 10, 2018

DOI 10.1212/WNL.0000000000006469

This information is current as of October 10, 2018

Neurology ${ }^{\circledR}$ is the official journal of the American Academy of Neurology. Published continuously since 1951, it is now a weekly with 48 issues per year. Copyright Copyright (C) 2018 The Author(s). Published by Wolters Kluwer Health, Inc. on behalf of the American Academy of Neurology.. All rights reserved. Print ISSN: 0028-3878. Online ISSN: 1526-632X.

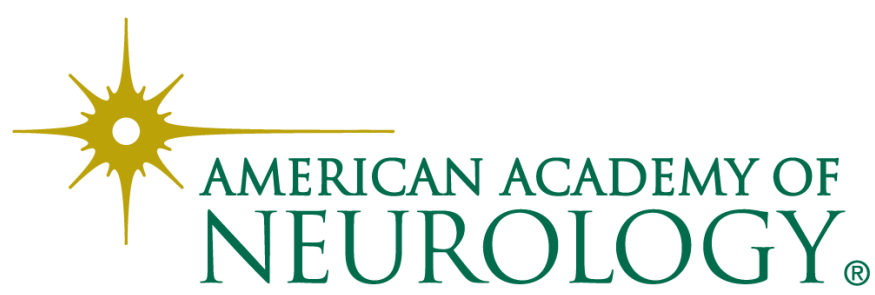




\section{Updated Information \& Services}

\section{Supplementary Material}

References

Citations

Subspecialty Collections

\section{Permissions \& Licensing}

Reprints including high resolution figures, can be found at: http://n.neurology.org/content/91/19/e1809.full

Supplementary material can be found at: http://n.neurology.org/content/suppl/2020/03/04/WNL.0000000000006 469.DC1

This article cites 44 articles, 12 of which you can access for free at: http://n.neurology.org/content/91/19/e1809.full\#ref-list-1

This article has been cited by 8 HighWire-hosted articles: http://n.neurology.org/content/91/19/e1809.full\#\#otherarticles

This article, along with others on similar topics, appears in the following collection(s):

\section{Alzheimer's disease}

http://n.neurology.org/cgi/collection/alzheimers_disease

\section{Cognitive aging}

http://n.neurology.org/cgi/collection/cognitive_aging

\section{Memory}

http://n.neurology.org/cgi/collection/memory

\section{PET}

http://n.neurology.org/cgi/collection/pet

Information about reproducing this article in parts (figures,tables) or in its entirety can be found online at:

http://www.neurology.org/about/about_the_journal\#permissions

Information about ordering reprints can be found online:

http://n.neurology.org/subscribers/advertise

Neurology ${ }^{\circledR}$ is the official journal of the American Academy of Neurology. Published continuously since 1951, it is now a weekly with 48 issues per year. Copyright Copyright ( 2018 The Author(s). Published by Wolters Kluwer Health, Inc. on behalf of the American Academy of Neurology.. All rights reserved. Print ISSN: 0028-3878. Online ISSN: 1526-632X.

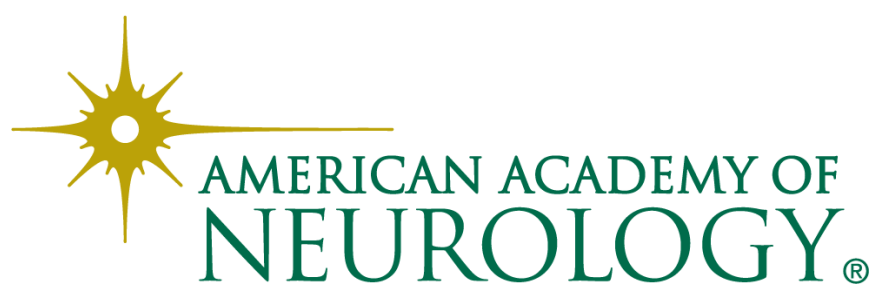

